\title{
Risk Factors for Disease Recurrence in Women With Phyllodes Tumors of the Breast in Southern Colombia: A Nine-Year Cohort Study
}

\author{
Justo Olaya $1,2,3$, Juan Sanjuan ${ }^{4,5,6,7}$, Rina L. Luna ${ }^{8}$, Lucia Casanova 9
}

1. Surgery - Mastology, Unidad Oncologica Surcolombiana, Neiva, COL 2. Surgery - Mastology, Universidad Surcolombiana, Neiva, COL 3. Surgery - Mastology, Hospital Universitario Hernando Moncaleano Perdomo, Neiva, COL 4. Clinical Research, Utopiapp SAS, Cali, COL 5. Clinical Research, Cirugia y Trauma (CYTRA) - Universidad Surcolombiana, Neiva, COL 6. Surgery, Universidad Surcolombiana, Neiva, COL 7. Surgery, Hospital Hernando Moncaleano Perdomo, Neiva, COL 8. Pathology, Hospital Hernando Moncaleano Perdomo, Neiva, COL 9. Cardiac Surgery, Clínica Medilaser, Neiva, COL

Corresponding author: Juan Sanjuan, drjuansanjuan@gmail.com

\section{Abstract}

\section{Introduction}

Phyllodes tumors (PTs) are uncommon fibroepithelial breast tumors that occur in middle-aged women, and they tend to vary in biologic behavior. Surgical management is the standard therapy for the condition, but factors associated with recurrence remain unclear. The aim of this study was to evaluate clinical and surgical characteristics related to PT recurrences.

\section{Methods}

This retrospective cohort study included patients in southern Colombia who were diagnosed with PT and managed at a level I teaching and referral hospital over a nine-year period. Factors associated with recurrence were determined by Cox regression analysis.

\section{Results}

This study included 61 patients; their median age was 46 years [interquartile range (IQR): 39-55 years]. Pathologically, 37 tumors (60.7\%) were classified as low-grade. The median tumor size was $7 \mathrm{~cm}$ (IQR: 4-11.5 $\mathrm{cm})$. Thirty-nine (63.9\%) patients underwent quadrantectomy. Nine patients (14.8\%) experienced tumor recurrence, with the median time to recurrence being one year (IQR: $0.5-2$ years). Distant metastasis was observed in four patients (6.6\%) at a median of nine months (IQR: 0.4-2.5 years). Univariate analyses showed that patients with high-grade tumors [hazard ratio $(\mathrm{HR}): 2.90, \mathrm{p}=0.148$ ] and those who underwent mastectomy (HR: $2.90, p=0.460$ ) were at higher risk of recurrence.

Received 04/23/2020

Review began 04/26/2020 Review ended 04/26/2020 Published 05/04/2020

\section{(c) Copyright 2020}

Olaya et al. This is an open access article distributed under the terms of the Creative Commons Attribution License CC-BY 4.0., which permits unrestricted use, distribution, and reproduction in any medium, provided the original author and source are credited.

\section{Conclusion}

PT recurrence may be associated with biological features, the extent of local excision, tumor size, and negative margins. However, multicenter data are needed to confirm these findings.

\begin{abstract}
Categories: General Surgery, Oncology, Other
Keywords: phyllodes tumors, mastology, breast disease, thoracic and breast oncology - areas of interest

\section{Introduction}

Phyllodes tumors (PTs) are rare fibroepithelial neoplasms that compromise the mammary glands. Estimates have indicated that around $0.3-1 \%$ of all breast tumors in women are PTs [1]. PTs are more prevalent in women aged 40-50 years than in other age groups [1-3]. The World Health Organization (WHO) has classified these tumors according to their behavior and biological characteristics as benign, borderline, or malignant $[4,5]$. These tumors have also been classified according to their invading ability and recurrence, the latter being either local or distant $[4,5]$. Surgical resection is the gold standard therapy, with margins of $\geqslant 1 \mathrm{~cm}$ reducing the risk of tumor recurrence [6,7]. Also, adjuvant radiotherapy is recommended under the principles for treating soft-tissue sarcomas [6,7]. Features of PTs associated with disease recurrence remain unclear [8]. The present study was designed to assess the relationships between tumor recurrence and the clinical and surgical characteristics of patients diagnosed with PT who were treated in southern Colombia over a nine-year period.
\end{abstract}

\section{Materials And Methods}

\section{Study features}


Patients with PT who were diagnosed and treated at a level I academic and referral hospital in southern Colombia over a nine-year period from 2007 to 2015 were retrospectively analyzed. The medical records of these patients were reviewed. The study protocol was approved by the local Institutional Review Board.

\section{Statistical analysis}

The sociodemographic characteristics, clinical evaluation, histopathological profile, management, disease recurrence, and mortality rate during follow-ups were analyzed. Categorical variables were summarized as absolute and relative frequencies. Continuous variables were reported as medians and interquartile ranges (IQRs). Recurrence was analyzed using the Kaplan-Meier method and compared using log-rank tests. The relationship between recurrence and clinical and surgical features was assessed by calculating hazard ratios (HRs) and 95\% confidence intervals (CIs). A p-value of $<0.05$ was considred statistically significant.

\section{Results}

Over the nine-year period, 61 patients of median age 46 years (IOR: 39-55 years) were diagnosed with PT. Tumors were located in the right mammary gland in 32 patients (52.5\%) and the left mammary gland in 29 patients (47.5\%). The median follow-up period was 61 months (IQR: 36-87 months). Histopathological analysis showed that 37 patients $(60.7 \%)$ had benign tumors, 15 (24.6\%) had borderline tumors, and nine (14.8\%) had malignant tumors. The median tumor size was $7 \mathrm{~cm}$ (IQR: 4-11.5 cm; range: 2-50 cm). Surgical management included wide local resection in three (4.9\%) patients, lumpectomy in 39 (63.9\%), and simple mastectomy in 19 (31.2\%). The three patients who underwent lumpectomy had positive surgical margins, with two of these patients having benign tumors and the third having a borderline tumor; therefore, their surgical margins were widened. Because of the persistence of compromised margins, one of these patients required a mastectomy.

Disease recurrence was observed in nine patients (14.8\%); the median time to recurrence was one year (IQR: 0.5-2 years; Figure 1). Five of these patients were diagnosed with benign tumors, one with a borderline tumor, and three with malignant tumors. The five patients diagnosed with benign tumors experienced local recurrence within a median time of approximately one year (IQR: 0.75-2 years; Figure 2). Initially, one patient underwent wide local resection, three underwent a lumpectomy, and one underwent a mastectomy due to a weak relationship between the mammary gland and the tumor (Table 1). The other four patients, one with a borderline tumor and three with malignant tumors, were diagnosed with distant recurrence and lung compromise at a median of eight months (IQR: 0.4-2.5 years; Table 2; Figure 3). All of these patients underwent a mastectomy.

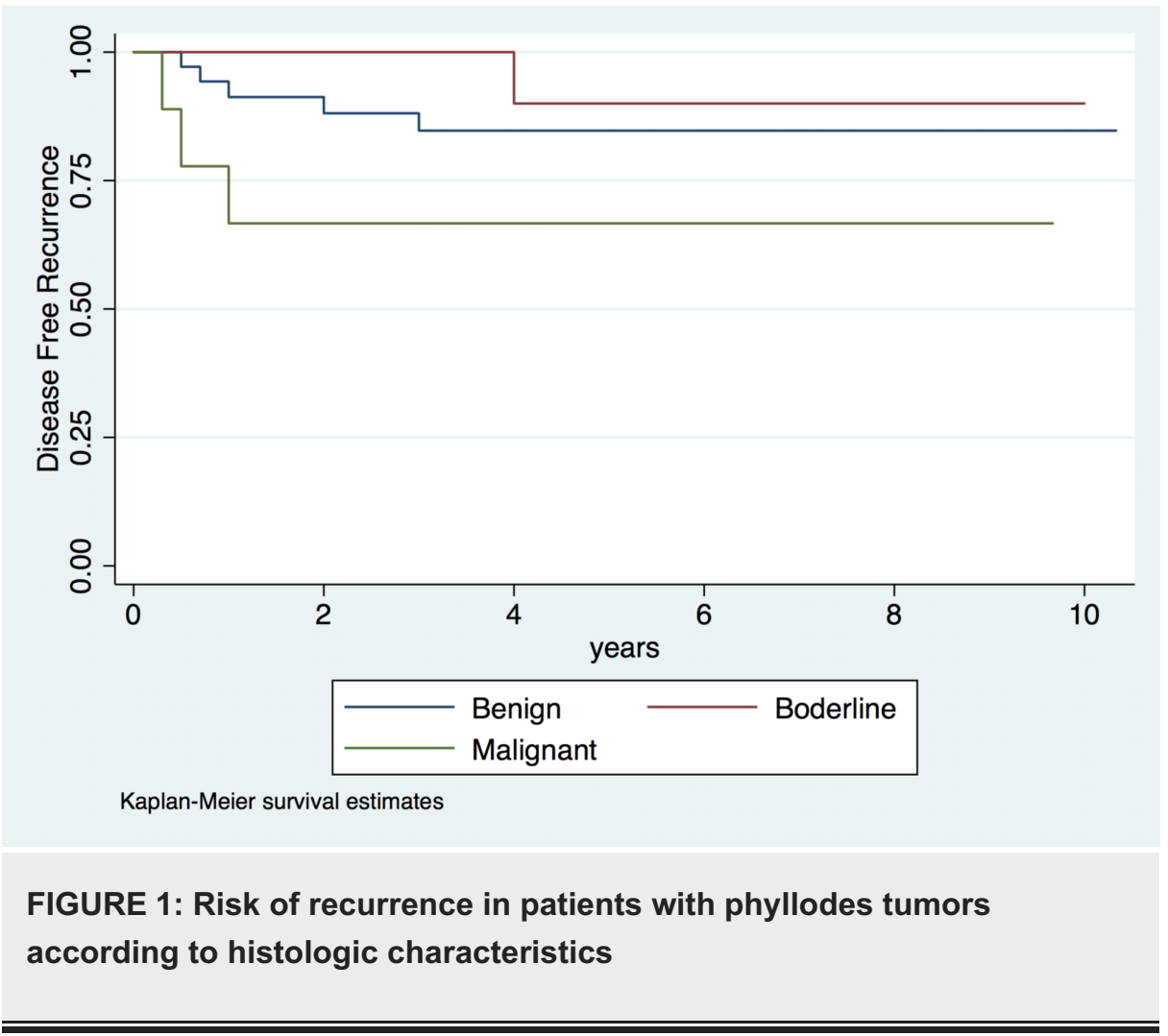




\section{Cureus}

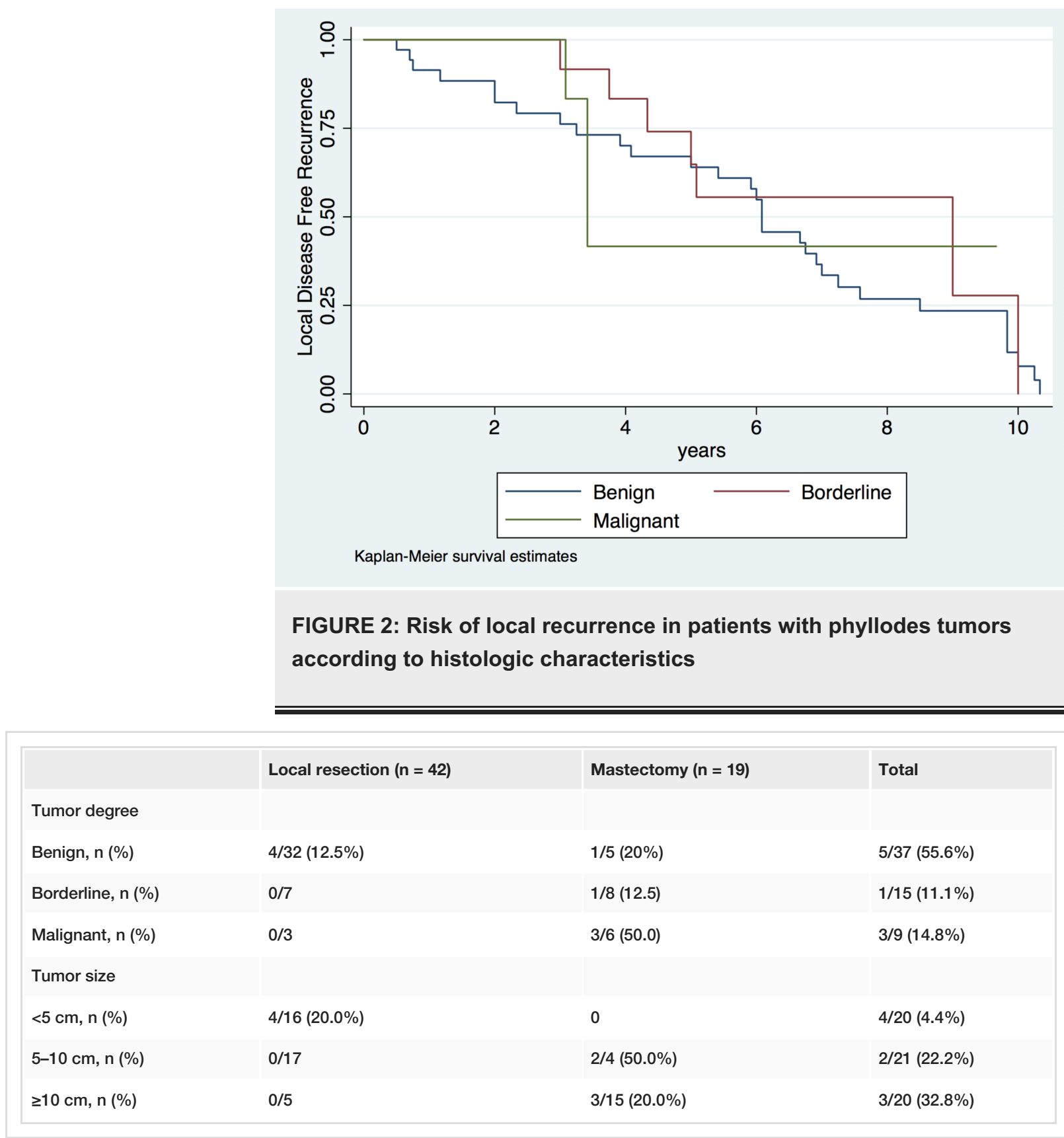

TABLE 1: Relationship of histologic degree and size of phyllodes tumors with surgical management and disease recurrence 


\section{Cureus}

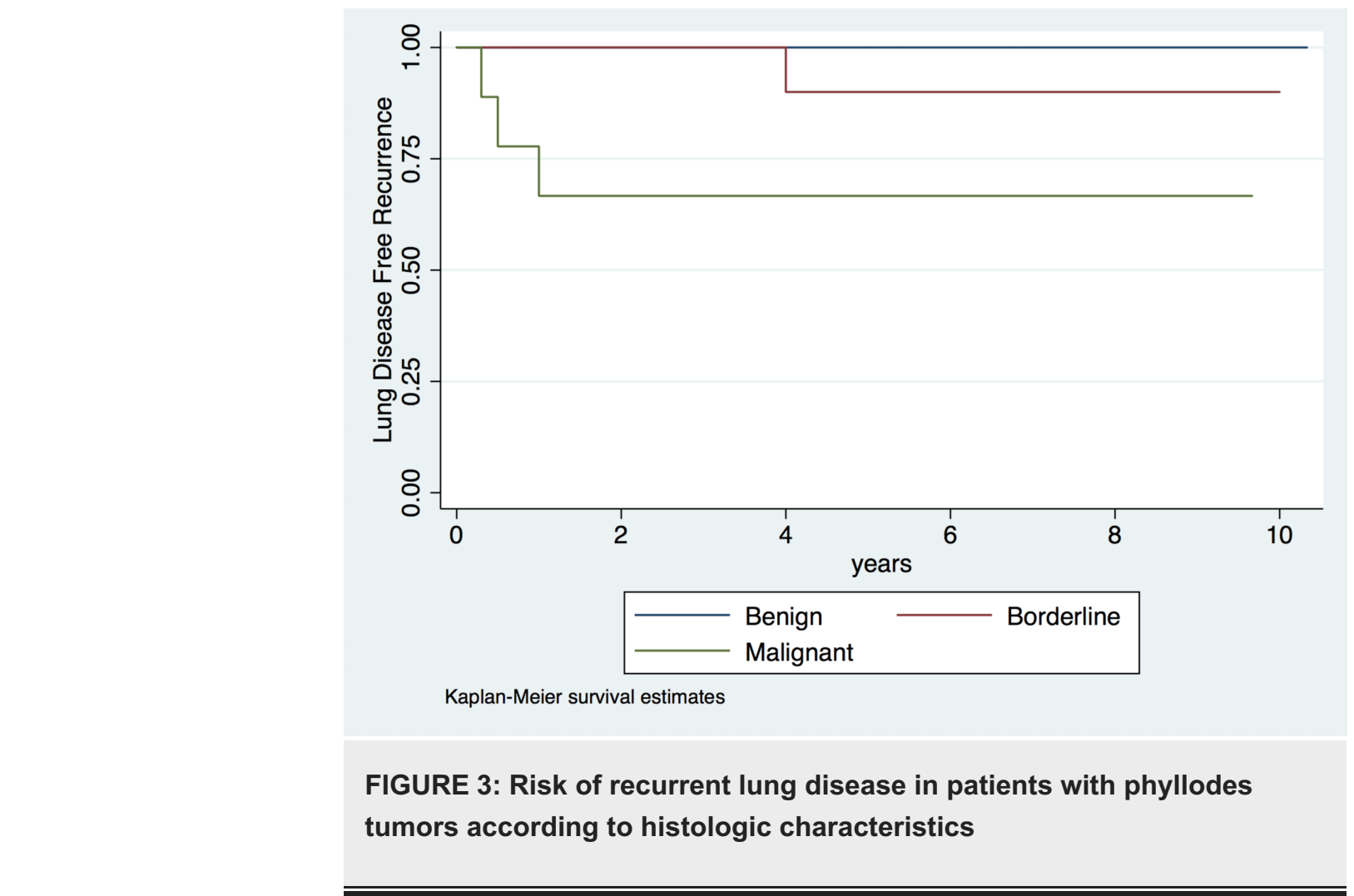

Univariate analysis results indicated that the risk of recurrence was higher in patients with malignant tumors (HR: 2.90; 95\% CI: 0.69-12.25, $\mathrm{p}=0.148$ ) and in those who underwent radiotherapy (HR: 9.05; 95\% CI: 2.42-33.8, $\mathrm{p}=0.001$; Table 2). The risk of recurrence tended to be higher in patients who underwent mastectomy (HR: 3.79; 95\% CI: 1.02-14.14, $\mathrm{p}=0.0 .47$ ) but was not associated with tumor size. Of the 61 patients, four (6.6\%) died, all with distant recurrence.

\begin{tabular}{|c|c|c|c|}
\hline & $H R$ & $95 \% \mathrm{Cl}$ & P-value \\
\hline Mastectomy & 3.79 & $1.02-14.14$ & 0.047 \\
\hline \multicolumn{4}{|l|}{ Tumor degree } \\
\hline Low & 1 & & \\
\hline Borderline & 0.51 & $0.6-4.33$ & 0.534 \\
\hline Malignant & 2.90 & $0.69-12.25$ & 0.148 \\
\hline \multicolumn{4}{|l|}{ Iumor size } \\
\hline$<5 \mathrm{~cm}$ & 1 & & \\
\hline $5-10 \mathrm{~cm}$ & 0.43 & $0.08-2.33$ & 0.325 \\
\hline$\geq 10 \mathrm{~cm}$ & 0.90 & $0.20-4.03$ & 0.889 \\
\hline Radiotherapy & 9.05 & $2.42-33.8$ & 0.001 \\
\hline
\end{tabular}

TABLE 2: Hazard ratio for recurrent disease in patients with phyllodes tumors following surgery

HR: hazard ratio; $\mathrm{Cl}$ : confidence interval

\section{Discussion}

The gold standard procedure for PTs consists of surgical management to control the wound and reduce recurrence [9]. Factors associated with disease recurrence may include biological behavior, tumor size, and surgical approach; however, the lack of randomized studies has prevented a definitive determination of 
factors associated with tumor recurrence $[3,9]$. The identification of such factors may guide treatment decisions and surgical approaches, resulting in better patient outcomes.

The present study analyzed patients with PTs who were treated at an academic and regional referral center in southern Colombia with an attention complexity level of III/IV. The medical histories of all patients were assessed to determine disease recurrence rates. Similar to previous reports, we found that the recurrence rate was $14.8 \%$, and the mortality rate was $6.6 \%[2,3,5,8]$. In line with other studies, we found that PTs were most prevalent in women in their fourth and fifth decades of life, although age was not a risk factor for disease recurrence $[2,10,11]$. Tumor sizes were larger than in previous reports, ranging up to $50 \mathrm{~cm}$, although we found that tumor size was not a significant risk factor for recurrence, even after adjusting for histological features $[2,11]$. In contrast, a previous study found that median tumor size was significantly greater in patients with recurrence compared to those without recurrence ( 8.8 vs. $5.4 \mathrm{~cm}, \mathrm{p}=<0.001$ ) [10].

In contrast to the distribution of histopathological categories of PT behavior, we found that the proportion of patients with benign tumors was higher than in other international studies $[2,10,12]$. Histological features have been reported to be associated with the probability of disease recurrence [1,11]. In our study, some patients with PTs classified as benign experienced local recurrence, with one undergoing lumpectomy due to the persistence of compromised margins after undergoing surgery to widen margins. Distant recurrence was observed in some patients with borderline and malignant PTs, all of whom were treated with lumpectomy. This distribution was comparable to that in other studies [10-13]. Although stromal overgrowth has also been associated with the risk of disease recurrence, this factor was not included in our study [3,11].

A meta-analysis reported that local recurrence is significantly associated with surgical treatment, specifically with mammary-gland conserving surgery in patients with malignant PTs (odds ratio: 2.32; 95\% CI: $1.01-5.30, \mathrm{p}=0.05$ ) [11]. However, the surgical approach has been based on histological features, the tumor-to-mammary-gland ratio, and patient desire for breast preservation, indicating that recurrence risk is biased by an aggressive surgical approach relative to the differential risk of disease recurrence. Compromised resection margins have also been linked to local and distant recurrence [14-17]. However, these rates were lower in our study than in previous reports $[2,3,8]$. Indeed, only one patient in the present study had compromised resection margins, and this patient experienced a local recurrence.

Similar to previous studies, we found that the association between radiotherapy and disease recurrence was statistically significant $(\mathrm{p}=0.001)$. Specific clinical and pathologic characteristics, including patient age, tumor size, type of surgery, and biological behavior may increase the likelihood of radiotherapy [2,18]. A recent meta-analysis suggested that the use of adjuvant therapy remains controversial, with the increased use of radiotherapy benefitting individual patients with PT, including younger women and patients with large-sized tumors, malignant histological behavior, and wide resection margins [18].

Our findings suggest that patients with malignant PTs are at higher risk of local and distant recurrence than patients with benign and borderline PTs. A nomogram illustrating the prognostic value and uncertainty of histological classification may provide a guide to the treatment of patients with PTs [8]. Stromal overgrowth should also be analyzed. The lack of inclusion of this feature in the current study is one of its main limitations. Another limitation was associated with the restricted analysis of subcategories due to the small sample size.

\section{Conclusions}

To contribute to the understanding of PTs, we assessed clinical and surgical characteristics related to PT recurrences. Despite systematic reviews, some features related to disease recurrence remain unclear. One consistent finding has been that tumor size does not relate to recurrence, unlike histological features and surgical and radiotherapy approaches. Some factors might be related to the differential risk of disease recurrence.

\section{Additional Information \\ Disclosures}

Human subjects: Consent was obtained by all participants in this study. Hospital Hernando Moncaleano Perdomo de Neiva IRB issued approval 005-002. This study was approved by the Hospital Hernando Moncaleano Perdomo de Neiva IRB with the approval number 005-002. Animal subjects: All authors have confirmed that this study did not involve animal subjects or tissue. Conflicts of interest: In compliance with the ICMJE uniform disclosure form, all authors declare the following: Payment/services info: All authors have declared that no financial support was received from any organization for the submitted work. Financial relationships: All authors have declared that they have no financial relationships at present or within the previous three years with any organizations that might have an interest in the submitted work. Other relationships: All authors have declared that there are no other relationships or activities that could appear to have influenced the submitted work.

\section{References}


1. Corso D, Contreras D, Javier Á, et al.: Tumor filoide. Estado del arte. (Article in Spanish). Rev Colomb Cancerol. 2016, 20:79-86. 10.1016/j.rccan.2015.10.001

2. Ossa CA, Herazo F, Gil M, et al.: Phyllodes tumor of the breast: a clinic-pathologic study of 77 cases in a Hispanic cohort. Colomb Medica (Cali). 2015, 46:104-8.

3. Asoglu O, Ugurlu MM, Blanchard K, Grant CS, Reynolds C, Cha SS, Donohue JH: Risk factors for recurrence and death after primary surgical treatment of malignant phyllodes tumors. Ann Surg Oncol. 2004, 11:10117. 10.1245/ASO.2004.02.001

4. Zhou ZR, Wang CC, Yang ZZ, Yu XL, Guo XM: Phyllodes tumors of the breast: diagnosis, treatment and prognostic factors related to recurrence. J Thorac Dis. 2016, 8:3361-8. 10.21037/jtd.2016.11.03

5. Mishra S, Tiwary S, Mishra M, Khanna AK: Phyllodes tumor of breast: a review article . ISRN Surg. 2013, 2013:361469. 10.1155/2013/361469

6. von Mehren M, Randall RL, Benjamin RS, et al.: Soft tissue sarcoma, version 2.2018: NCCN Clinical Practice Guidelines in Oncology. J Natl Compr Canc Netw. 2018, 16:536-63. 10.6004/jnccn.2018.0025

7. NCCN Clinical Practice Guidelines in Oncology: breast cancer - version 1.2018 . (2018). Accessed: May 3, 2020: https://www2.tri-kobe.org/nccn/guideline/archive/breast2018/english/breast_v1.pdf.

8. Zhou ZR, Wang CC, Sun XJ, et al.: Prognostic factors in breast phyllodes tumors: a nomogram based on a retrospective cohort study of 404 patients. Cancer Med. 2018, 7:1030-42. Accessed: May 3, 2020: https://www.ncbi.nlm.nih.gov/pmc/articles/PMC5911599/. 10.1002/cam4.1327

9. Adesoye T, Neuman HB, Wilke LG, Schumacher JR, Steiman J, Greenberg CC: Current trends in the management of phyllodes tumors of the breast. Ann Surg Oncol. 2016, 23:3199-205. 10.1245/s10434-0165314-0

10. Jang JH, Choi MY, Lee SK, et al.: Clinicopathologic risk factors for the local recurrence of phyllodes tumors of the breast. Ann Surg Oncol. 2012, 19:2612-7. 10.1245/s10434-012-2307-5

11. Lu Y, Chen Y, Zhu L, Cartwright P, Song E, Jacobs L, Chen K: Local recurrence of benign, borderline, and malignant phyllodes tumors of the breast: a systematic review and meta-analysis. Ann Surg Oncol. 2019, 26:1263-75. 10.1245/s10434-018-07134-5

12. Wei J, Tan YT, Cai YC, et al.: Predictive factors for the local recurrence and distant metastasis of phyllodes tumors of the breast: a retrospective analysis of 192 cases at a single center. Chin J Cancer. 2014, 33:492500. 10.5732/cjc.014.10048

13. Koh VCY, Thike AA, Tan PH: Distant metastases in phyllodes tumours of the breast: an overview . Appl Cancer Res. 2017, 37:15. Accessed: May 3, 2020:

https://appliedcr.biomedcentral.com/articles/10.1186/s41241-017-0028-6. 10.1186/s41241-017-0028-6

14. Huang W, Altaf K, Jin T, et al.: Prediction of the severity of acute pancreatitis on admission by urinary trypsinogen activation peptide: a meta-analysis. World J Gastroenterol. 2013, 19:4607-15. 10.3748/wjg.v19.i28.4607

15. Abdelkrim SB, Trabelsi A, Bouzrara M, Boudagga MZ, Memmi A, Bakir DA, Mokni M: Phyllodes tumors of the breast: a review of 26 cases. World J Oncol. 2010, 1:129-34. 10.4021/wjon2010.06.220w

16. Majeski J, Stroud J: Malignant phyllodes tumors of the breast: a study in clinical practice . Int Surg. 2012, 97:95-8. 10.9738/CC79.1

17. Ben Hassouna J, Damak T, Gamoudi A, et al.: Phyllodes tumors of the breast: a case series of 106 patients . Am J Surg. 2006, 192:141-7. 10.1016/j.amjsurg.2006.04.007

18. Gnerlich JL, Williams RT, Yao K, Jaskowiak N, Kulkarni SA: Utilization of radiotherapy for malignant phyllodes tumors: analysis of the National Cancer Data Base, 1998-2009. Ann Surg Oncol. 2014, 21:1222-30. 10.1245/s10434-013-3395-6 